\title{
PREVALENCIA DEL RIESGO DE TRASTORNOS DE LA CONDUCTA ALIMENTARIA EN UNA POBLACIÓN DE ESTUDIANTES DE SECUNDARIA, BOGOTÁ - COLOMBIA
}

\author{
ESPERANZA FAJARDO ${ }^{1}$, CAROLINA MÉNDEZ ${ }^{2}$, ALFONSO JAUREGUI $^{3}$ \\ ${ }^{1} \mathrm{ND}, \mathrm{MSc}$. Directora Grupo de Investigación CATCH. \\ Facultad de Medicina. Universidad Militar Nueva Granada. Bogotá-Colombia \\ ${ }^{2} \mathrm{ND}, \mathrm{MSc}$. Asistente de Investigación Grupo de Investigación CATCH. Facultad de Medicina. \\ Universidad Militar Nueva Granada. Bogotá-Colombia \\ ${ }^{3}$ Médico Internista. Miembro Grupo de Investigación CATCH. Facultad de Medicina. \\ Universidad Militar Nueva Granada. Bogotá-Colombia \\ *Correspondencia: esperanza.fajardo@unimilitar.edu.co
}

Recibido: 7 Diciembre $2016 \quad$ Aceptado: 3 Abril 2017

\section{RESUMEN}

Objetivo: Describir la prevalencia de riesgo de trastornos de la conducta alimentaria, estado nutricional y consumo de alimentos en una población escolar de secundaria de colegios públicos y privados.

Materiales y Métodos: Aplicación del cuestionario SCOFF, toma de medidas antropométricas y encuesta de frecuencia de consumo de alimentos a 671 estudiantes de dos colegios públicos y dos privados de la ciudad de Bogotá.

Resultados: Se encontró una prevalencia del 30,1\% de riesgo de presentar TCA; de ésta, el riesgo en las mujeres es de $41,3 \%$. En cuanto al estado nutricional se observó mayor frecuencia de riesgo en la clasificación normal y en instituciones públicas con un $31,3 \%$. Del total de la población 7,6\% presenta talla baja, $16 \%$ exceso de peso y $5,1 \%$ bajo.

Conclusiones: La prevalencia de riesgo de trastornos alimentarios es mayor en las mujeres, con clasificación nutricional normal $(73,7 \%)$ y en instituciones educacionales públicas.

Palabras clave: Cuestionario SCOFF; Estudiantes; Conducta alimentaria; Estado Nutricional 


\title{
PREVALENCE OF EATING DISORDERS RISK IN A POPULATION OF HIGH SCHOOL STUDENTS, BOGOTÁ - COLOMBIA
}

\begin{abstract}
Objective: To describe the prevalence of eating disorders risk, nutritional status and food consumption in a high school population of public and private schools.

Materials and Methods: The SCOFF questionnaire application, anthropometric measurements and food consumption frequency survey to 671 students from two public and two private schools in the city of Bogotá.

Results: A prevalence of $30.1 \%$ risk of presenting ACT was found; of that, the risk in women is $41.3 \%$. Regarding nutritional status, there was a greater frequency of risk in the normal classification and in public institutions with $31.3 \% .7 .6 \%$ of the total population were of low stature, $16 \%$ were overweight and $5.1 \%$ were underweight.
\end{abstract}

Conclusions: Prevalence of eating disorders risk is higher in women, with normal nutritional status $(73.7 \%)$ and in public educational institutions.

Key words: SCOFF questionnaire; Students; Feeding behavior; Nutritional Status

\section{PREVALÊNCIA DE RISCO DE TRASTORNOS DO COMPORTAMENTO ALIMENTAR EM POPULAÇÃO DE ESTUDANTES DO ENSINO MEDIO, BOGOTÁ - COLOMBIA}

\section{RESUMO}

Objetivo: Descrever a prevalência de risco de transtornos do comportamento alimentar, estado nutricional e consumo de alimentos em uma população de estudantes do ensino médio de escolas públicas e privadas.

Materiais e métodos: A aplicação do questionário SCOFF, medidas antropométricas e levantamento de freqüência de consumo de alimentos para 671 estudantes de duas escolas públicas e duas privadas da cidade de Bogotá.

Resultados: Foi encontrada uma prevalência de 30,1\% de risco de apresentação do ACT; Disso, o risco em mulheres é de 41,3\%. Quanto ao estado nutricional, houve uma maior freqüência de risco na classificação normal e em instituições públicas com $31,3 \%$. $7,6 \%$ da população total eram de baixa estatura, $16 \%$ tinham sobrepeso e $5,1 \%$ estavam abaixo do peso.

Conclusões: A prevalência do risco de transtorno alimentar é maior em mulheres, com estado nutricional normal $(73,7 \%)$ e em instituições públicas de ensino.

Palavras-chave: Questionário SCOFF; Estudantes; Comportamento de alimentação; Estado nutricional. 


\section{Introducción}

Los trastornos del comportamiento alimentario (TCA), como son la Anorexia Nerviosa (AN), Bulimia Nerviosa (BN) y los trastornos del comportamiento alimentario no especificados (TCANE) son el resultado de la interacción de factores psicológicos, físicos y socio-culturales que interfieren en el comportamiento de los individuos especialmente en la población adolescente, etapa de la vida caracterizada por grandes cambios biológicos y psicosociales que generan un ambiente propicio para el desarrollo de estos trastornos produciendo consecuencias potencialmente graves para la salud de los jóvenes, su entorno familiar y social (1).

Éstas son enfermedades psiquiátricas graves, que se caracterizan por alteraciones en el comportamiento, las actitudes y la ingestión de alimentos generalmente acompañadas de intensa preocupación con el peso o con la forma del cuerpo (1). Su tratamiento es complejo y pueden llegar a ser perjudiciales para la salud y la nutrición; por otro lado, predispone a las personas a la desnutrición o a la obesidad (2) y se asocian con baja calidad de vida, altas tasas de comorbilidad psicosocial y mortalidad prematura (3).

Se suele relacionar su incremento con las exigencias sociales acerca de la apariencia del cuerpo, que en las últimas décadas presentan como modelo de éxito la delgadez extrema y la apariencia prepuberal, dándole un significado de éxito y triunfo a este tipo de imagen. No estar satisfecho con el cuerpo predispone a los jóvenes al uso de estrategias extremas como las dietas restrictivas, el ayuno, uso de laxantes y diuréticos, para lograr pérdida de peso con la idea de lograr beneficios físicos y psicosociales. Sin embargo, diversos estudios longitudinales han demostrado que las dietas restrictivas incrementan el riesgo de aumento de peso corporal lo que predispone a otros problemas de salud (4).

Un fenómeno que preocupa actualmente es el aumento de casos de TCA a edades más tempranas. La edad de aparición ha disminuido en 12 meses (5-8). En su mayoría, las adolescentes y adultas jóvenes están entre los grupos con mayor prevalencia de los TCA (9), aunque la ocurrencia no es despreciable entre los adolescentes varones (7).

Se ha descrito que, dentro de las razones para un incremento en la frecuencia de los trastornos de la conducta alimentaria en niños y jóvenes, es el comportamiento de los padres quienes ejercen un excesivo control sobre la alimentación de sus hijos, aspecto que sumado a la presión social y la de los amigos para que sean delgados, contribuyen al desarrollo de una imagen corporal negativa y por consiguiente a conductas alimentarias inadecuadas que ponen en riesgo la salud (4).

Estudios realizados en Colombia en población universitaria han mostrado prevalencia de TCA que oscilan entre el $18,9 \%$ y $39,7 \%$, siendo más frecuente la bulimia nerviosa que la anorexia nerviosa, entre mujeres adolescentes y adultos jóvenes (10 -14).

En el 2005 Rueda et al. (10) validaron el cuestionario SCOFF, acrónimo de las iniciales de las preguntas de cada ítem en inglés: Inducirse vómito (sick), control sobre la ingesta (control), pérdida de peso (out weight), sentirse gordo (fat) y la comida domina la vida (food) (15), en estudiantes universitarias y de secundaria con aceptables pruebas de desempeño. El cuestionario SCOFF tiene ventajas por la brevedad del instrumento para tamizaje en estudios con grandes poblaciones (16).

Numerosos han sido los estudios e intervenciones sobre los trastornos del comportamiento alimentario. Resulta interesante entonces, identificar precozmente tanto alteraciones del peso corporal como de la conducta alimentaria con miras a diseñar estrategias de prevención y control que puedan estandarizarse y aplicarse en niños y jóvenes contribuyendo de esta manera en un mejor estado de salud de nuestra sociedad.

En este estudio se busca identificar la prevalencia de riesgo de trastornos de la conducta alimentaria, el estado nutricional y el consumo de alimentos en una población de estudiantes de los grados $6^{\circ}$ a $11^{\circ}$ de colegios públicos y privados de la ciudad de Bogotá, con el fin de proponer medidas de intervención para contrarrestar estos problemas nutricionales, estrategias que servirán para continuar con la línea de investigación.

\section{Materiales y Métodos}

Se realizó un estudio descriptivo de corte transversal con componente analítico.

\section{Población y muestra}

La muestra estuvo conformada por 671 estudiantes de secundaria entre los 10 a 20 años matriculados en dos colegios públicos y dos privados seleccionados por conveniencia de la ciudad de Bogotá. 


\section{Criterios de inclusión}

Se consideraron elegibles todos los estudiantes de educación básica secundaria de $6^{\circ}$ a $11^{\circ}$ grado que se encuentran matriculados en los colegios seleccionados.

\section{Criterios de exclusión}

Fueron excluidos los estudiantes que no desearon participar o cuyos padres no aceptaron la participación en el estudio, o quienes no diligenciaron el consentimiento $y / o$ asentimiento informado.

El estudio contó con la autorización de los directivos y la colaboración de los profesores de cada una de las instituciones educativas. Se impartió información a los participantes y a sus padres sobre las características del estudio y el procedimiento; posteriormente se distribuyó el formato del consentimiento informado para firma de los padres del estudiante y el estudiante, acorde a la decisión libre de cada uno de ellos.

\section{Procedimiento}

En las instalaciones de cada uno de los colegios, se realizó un estudio antropométrico tomando estatura, peso y circunferencia de cintura a cada uno de los estudiantes participantes del estudio.

Se entregó una encuesta que incluía los antecedentes nutricionales y alimentarios, así como la Frecuencia de Consumo de Alimentos que constituye un método directo de estimación de la ingesta alimentaria de un individuo a partir de un formulario estructurado donde el encuestado responde el número de veces que consume en promedio un alimento durante un periodo de tiempo determinado. El instrumento se encuentra validado y permite obtener a partir de un listado de alimentos, la frecuencia habitual de ingesta de un alimento durante una semana.

\section{Técnicas e instrumentos}

El registro de las medidas antropométricas se realizó en el programa Microsoft Office Excel 2011.

Para la toma de peso y estatura se utilizó una báscula de peso corporal electrónica (Tanita, capacidad $150 \mathrm{~kg}$, precisión $\pm 1 \%+0.1 \mathrm{~kg}$ ), tallímetro y cinta métrica. Estas medidas se tomaron sin zapatos y con el mínimo de ropa posible. La estatura se tomó con los pies y las rodillas juntas, talones, cara posterior de glúteos y cabeza bien adheridos al plano del tallímetro, se colocó una escuadra sobre el vértex y se leyó el valor de la talla en centímetros.
La circunferencia de la cintura se tomó al final de una expiración normal, a un nivel intermedio entre el último arco costal y la cresta ilíaca, en la posición más estrecha del abdomen.

El índice de masa corporal (IMC) se construyó a partir de los datos de peso y talla (peso en $\mathrm{kg} /$ talla $\mathrm{m}^{2}$ ). Para la clasificación nutricional, se utilizaron las tablas de indice de masa corporal por edad, para hombres y mujeres de 2 a 20 años establecidas por el Centro de Estadísticas de Salud de Estados Unidos (USA National Center for Health Statistics). Los valores de indice de masa corporal menores del percentil 5 son considerados como bajo peso; normal entre el percentil 5 y menos del 85; sobrepeso entre el percentil 85 y menos del 95 y obesidad en el percentil 95 o más.

Al mismo tiempo, se aplicó a los participantes un cuestionario que incluye datos socio-demográficos y el cuestionario SCOFF (15) (Tabla 1), para evaluar el riesgo de los trastornos de la conducta alimentaria. Si se respondió afirmativamente a dos o más de las preguntas sobre el comportamiento, es probable que curse con un trastorno conductual y debe ser manejado por el profesional de la salud.

Tabla 1. Versión del cuestionario SCOFF.

Morgan JF, Reid F, Lacey JH. The SCOFF questionnaire: assessment of a new screening tool for eating disorder. $\mathrm{Br}$ Med J 1999; 319: 1467-8.

\begin{tabular}{|l|l|}
\hline & PREGUNTA \\
\hline $\mathbf{1 .}$ & $\begin{array}{l}\text { ¿Tiene la sensación de estar enfermo(a) porque } \\
\text { siente el estómago tan lleno que le resulta } \\
\text { incómodo? }\end{array}$ \\
\hline $\mathbf{2 .}$ & $\begin{array}{l}\text { ¿Está preocupado(a) porque siente que tiene } \\
\text { que controlar cuanto come? }\end{array}$ \\
\hline $\mathbf{3 .}$ & $\begin{array}{l}\text { ¿Ha perdido recientemente más de } 6 \text { kilos en } \\
\text { un período de tres meses? }\end{array}$ \\
\hline $\mathbf{4 .}$ & $\begin{array}{l}\text { ¿Cree estar gordo(a) aunque otros digan que } \\
\text { está delgado(a)? }\end{array}$ \\
\hline $\mathbf{5 .}$ & ¿Diría que la comida domina su vida? \\
\hline
\end{tabular}




\section{Análisis de datos}

Se realizó análisis univariado a través de tablas de distribución de frecuencias para variables cualitativas y medidas descriptivas de tendencia central, de posición y de dispersión para variables cuantitativas. El análisis bivariado y multivariado se realizó a través de tablas de contingencia. Para el análisis de los datos se utilizó SPSS versión 20.0 (SPSS Inc. Chicago, Illinois USA).

\section{Resultados}

Participaron 671 estudiantes, 359 (53,5\%) hombres y $312(46,5 \%)$ mujeres, con edades entre 10 a 20 años, promedio 14,05 años (D.E. $=2,06$ ). El $53,8 \%$ asiste a colegios públicos y $46,1 \%$ a privados. La distribución por sexo, tipo de establecimiento y rango de edad se presenta en la tabla 2.

Tabla 2. Distribución de la muestra por sexo y rango de edad según tipo de colegio.

\begin{tabular}{|l|c|c|c|c|c|c|c|}
\hline \multirow{2}{*}{ Colegio } & \multicolumn{2}{|c|}{$\mathbf{1 0 - 1 2}$ años } & \multicolumn{2}{c|}{$\mathbf{1 3 - 1 5}$ años } & \multicolumn{2}{c|}{ 16 o más años } & \multirow{2}{*}{ Total } \\
\cline { 2 - 8 } Público & H & M & H & M & H & M & \\
\hline Privado & 46 & 28 & 95 & 56 & 50 & 35 & 361 \\
\hline Total & 47 & 48 & 73 & 95 & 48 & 50 & 310 \\
\hline Total por grupo de edad & 93 & 76 & 168 & 151 & 98 & 85 & \\
\hline
\end{tabular}

Fuente: Elaboración propia

\section{Antecedentes nutricionales y alimentarios}

El $92.9 \%$ de escolares viven con sus padres o algún familiar. Un $84,8 \%$ de escolares consume alimentos en su hogar y un $66,5 \%$ los ingiere en compañía de sus fami- liares. En cuanto al tiempo empleado para consumir los alimentos el $47,2 \%$ de todos los escolares dedican entre 11 a 20 minutos. La tabla 3 muestra que el 12,2\% del total de estudiantes no desayuna y el $17,4 \%$ no acostumbra cenar.

Tabla 3. Frecuencia de consumo de comidas principales.

\begin{tabular}{|l|c|c|c|c|c|c|}
\hline \multirow{2}{*}{ Frecuencia } & \multicolumn{2}{|c|}{ Desayuno } & \multicolumn{2}{c|}{ Almuerzo } & \multicolumn{2}{c|}{ Cena } \\
\cline { 2 - 7 } & $\mathbf{n}$ & $\mathbf{\%}$ & $\mathbf{n}$ & $\mathbf{\%}$ & $\mathbf{n}$ & $\mathbf{\%}$ \\
\hline SI & 588 & 87,6 & 638 & 95,1 & 546 & 81,4 \\
\hline NO & 82 & 12,2 & 32 & 4,8 & 117 & 17,4 \\
\hline N/R & 1 & 0,1 & 1 & 0,1 & 8 & 1,2 \\
\hline Total & 671 & 100 & 671 & 100 & 671 & 100 \\
\hline
\end{tabular}

Fuente: Elaboración propia

El 69\% de los estudiantes que no acostumbra a desayunar refiere que es por "falta de tiempo". La principal razón para no cenar es "no le gusta" o "no acostumbra" en un $73,5 \%$ y el $21,9 \%$ por "falta de tiempo".
El $65,7 \%$ de los escolares refiere tres o más síntomas cuando deja de consumir alimentos en el día, como son dolor de cabeza, mareo, debilidad y dolor de estómago. 


\section{Frecuencia de consumo de alimentos}

El dato obtenido mediante el cuestionario de Frecuencia de Consumo de alimentos muestra que el $37,3 \%$ de los escolares no consume leche diariamente; en cuanto al consumo de carnes, que son fuente de proteína de alto valor biológico, el $82,1 \%$ de los encuestados no refiere consumo diario y aproximadamente la mitad de los escolares consumen huevo todos los días. El 53,8\% consume carne roja y pollo 2 a 3 veces por semana. Se observa un bajo consumo de pescado. El consumo diario de frutas enteras fue reportado por el $43 \%$ de los escolares y el $41,6 \%$ consume verduras diariamente; (Gráfico 1).

Gráfico 1. Frecuencia diaria de consumo por grupo de alimentos.

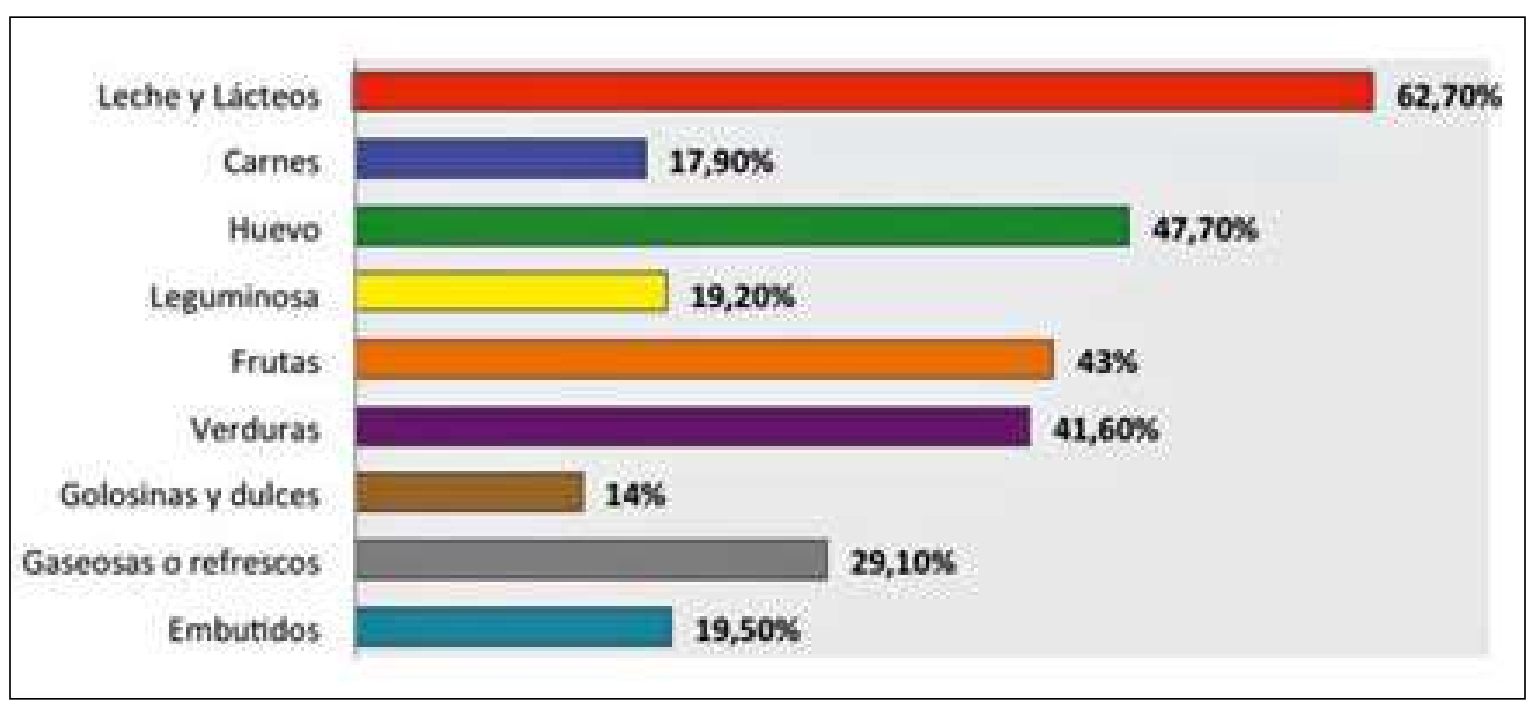

Fuente: Elaboración propia

Se reportó que ocasionalmente un $40,7 \%$ de escolares consume dulces y golosinas. Las gaseosas y refrescos se consumen 2 a 3 veces por semana en un $30,8 \%$ de la población de escolares. El $66,3 \%$ de los escolares reportó que el consumo de fruta lo realizan en forma de jugos naturales.

\section{Características Antropométricas de la Población}

En la Tabla 4 se presenta la clasificación nutricional según el IMC por sexo y tipo de institución.

Tabla 4. Clasificación nutricional de la población por índice de masa corporal según sexo y tipo de institución escolar.

\begin{tabular}{|l|c|c|c|c|c|c|}
\hline \multirow{2}{*}{$\begin{array}{c}\text { Clasificación } \\
\text { (IMC) }\end{array}$} & \multicolumn{2}{|c|}{ Privado } & \multicolumn{2}{c|}{ Público } & \multirow{2}{*}{ Total } & \multirow{2}{*}{$\%$} \\
\cline { 2 - 6 } & Niño (n) & Niña (n) & Niño (n) & Niña (n) & & \\
\hline Normal & 152 & 89 & 138 & 157 & 537 & 80 \\
\hline Sobrepeso & 25 & 19 & 13 & 26 & 83 & 12,3 \\
\hline Obesidad & 7 & 5 & 7 & 5 & 24 & 3,6 \\
\hline Bajo peso & 7 & 6 & 10 & 5 & 28 & 4,2 \\
\hline Total & 191 & 119 & 168 & 193 & 671 & 100 \\
\hline
\end{tabular}

Fuente: Elaboración propia 
Se observa un IMC normal en el $80 \%$ de los estudiantes; mayor porcentaje de bajo peso y obesidad en los hombres y en las mujeres es mayor el porcentaje de sobrepeso que en los hombres. Entre otros resultados, se encontró que el 7,6\% de la población total presenta talla baja según el indicador de talla para la edad. Teniendo en cuenta la prevalencia de sobrepeso y obesidad se encontró en la población un $16 \%$ de exceso de peso y $4,2 \%$ de bajo peso; (Gráfico 2).

Gráfico 2. Clasificación Nutricional por IMC.

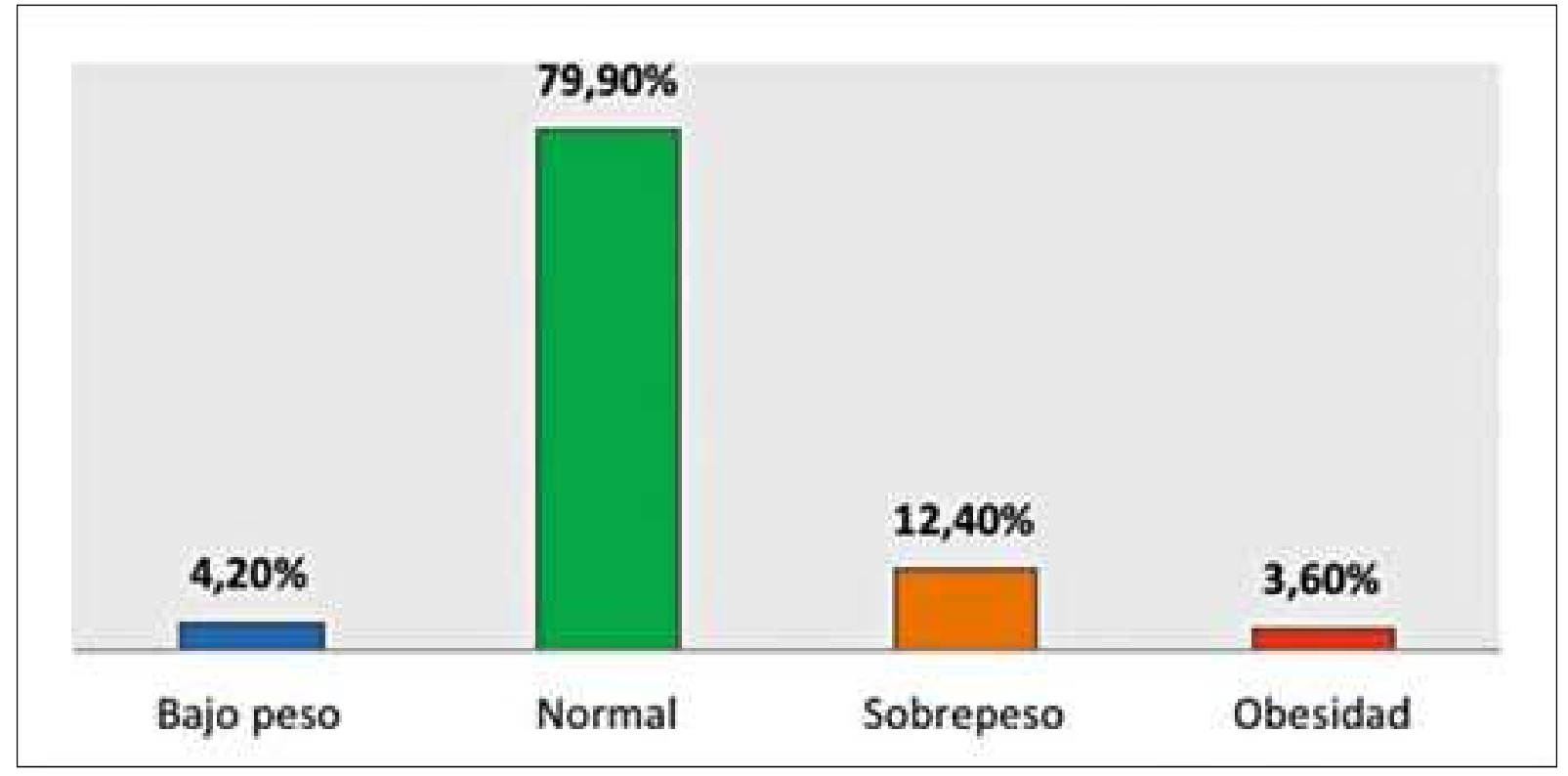

Fuente: Elaboración propia

En la población total según la Razón Cintura/Estatura (RCE) el 18,3\% presenta aumento en la grasa a nivel superior del cuerpo y es más frecuente en las mujeres (Tabla 5).

Tabla 5. Grasa a nivel superior del cuerpo según Razón Cintura/Estatura (RCE) de la población total y por sexo.

\begin{tabular}{|c|c|c|c|c|c|c|}
\hline \multirow{2}{*}{ RCE } & \multirow{2}{*}{ Frecuencia } & \multirow{2}{*}{ \% } & \multicolumn{2}{c|}{ Niño } & \multicolumn{2}{c|}{ Niña } \\
\cline { 4 - 7 } & & $\mathbf{n}$ & $\mathbf{\%}$ & $\mathbf{n}$ & $\mathbf{\%}$ \\
\hline Aumentada (>5) & 123 & 18,3 & 56 & 15,6 & 67 & 21,5 \\
\hline Adecuada (55) & 546 & 81,4 & 301 & 83,8 & 245 & 78,5 \\
\hline Sin dato & 2 & 0,3 & 2 & 0,4 & 0 & 0 \\
\hline \multicolumn{1}{c}{ Total } & 671 & 100 & 359 & 100 & 312 & 100 \\
\hline
\end{tabular}

Fuente: Elaboración propia

Al relacionar la clasificación nutricional según el índice de masa corporal con el aumento de grasa abdominal medido por RCE mayor de 0,5 , que se relaciona con riesgo cardiometabólico, se observó que los estudiantes con sobrepeso presentan un $44,7 \%$ de riesgo para este tipo de enfermedades. El 35,8\% de los estudiantes con riesgo tienen peso normal; (Gráfico 3). 
Gráfico 3. Porcentaje de estudiantes con riesgo cardiovascular según RCE de la población total por estado nutricional.

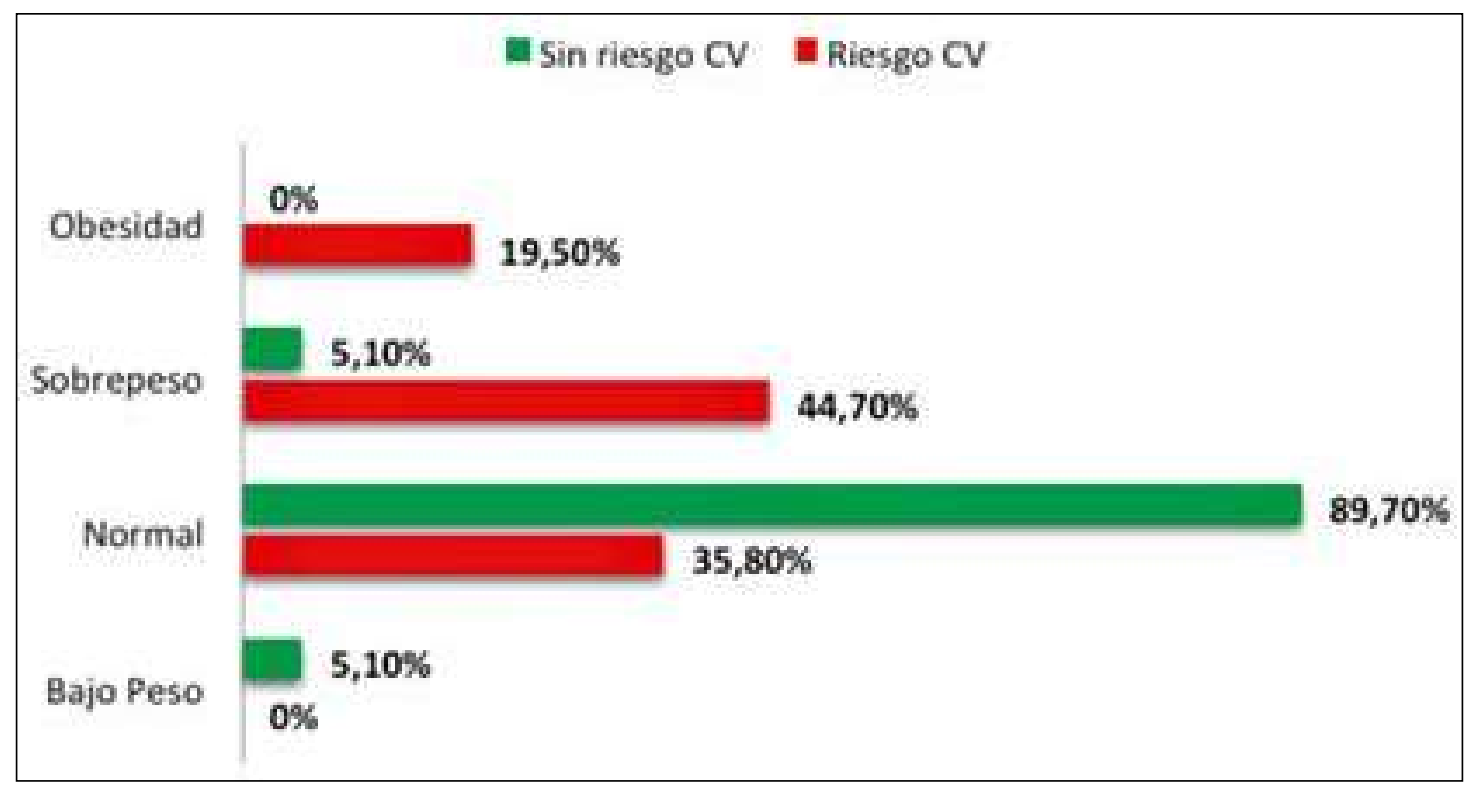

Fuente: Elaboración propia

\section{Frecuencia de Riesgo de Trastornos de la Conducta Alimentaria}

A nivel de la muestra total y mediante la utilización del test de SCOFF, se encontró que 202 participantes tienen riesgo de trastornos de la conducta alimentaria (TCA) $(30,1 \%)$. Al estratificar por sexo, se observó que el porcentaje de sujetos con este riesgo es mayor en mujeres que en varones; (Gráfico 4).

Gráfico 4. Porcentaje de escolares con riesgo de TCA por sexo

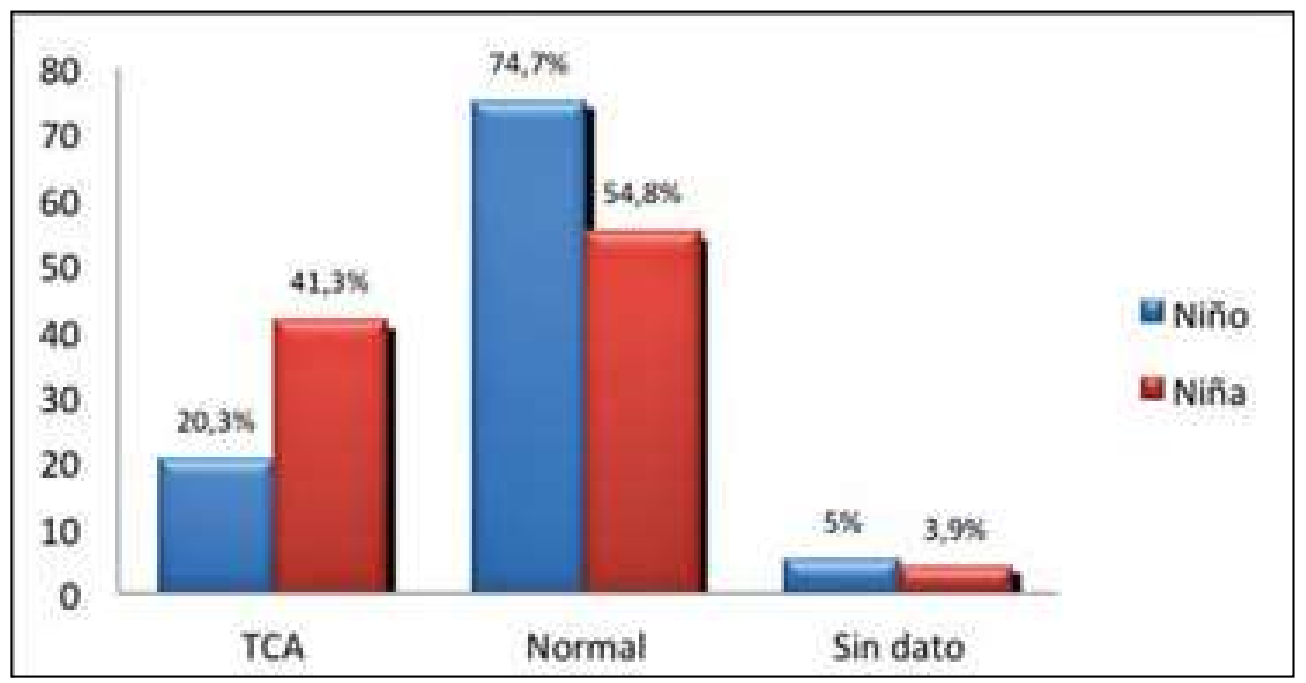

Fuente: Elaboración propia 
Al comparar el riesgo de TCA con el estado nutricional se observa que la mayoría de los casos se encuentra en los escolares con estado nutricional normal en un $73,7 \%$ (Gráfico 5))

Gráfico 5. Porcentaje de escolares con riesgo de TCA de acuerdo al Estado Nutricional.

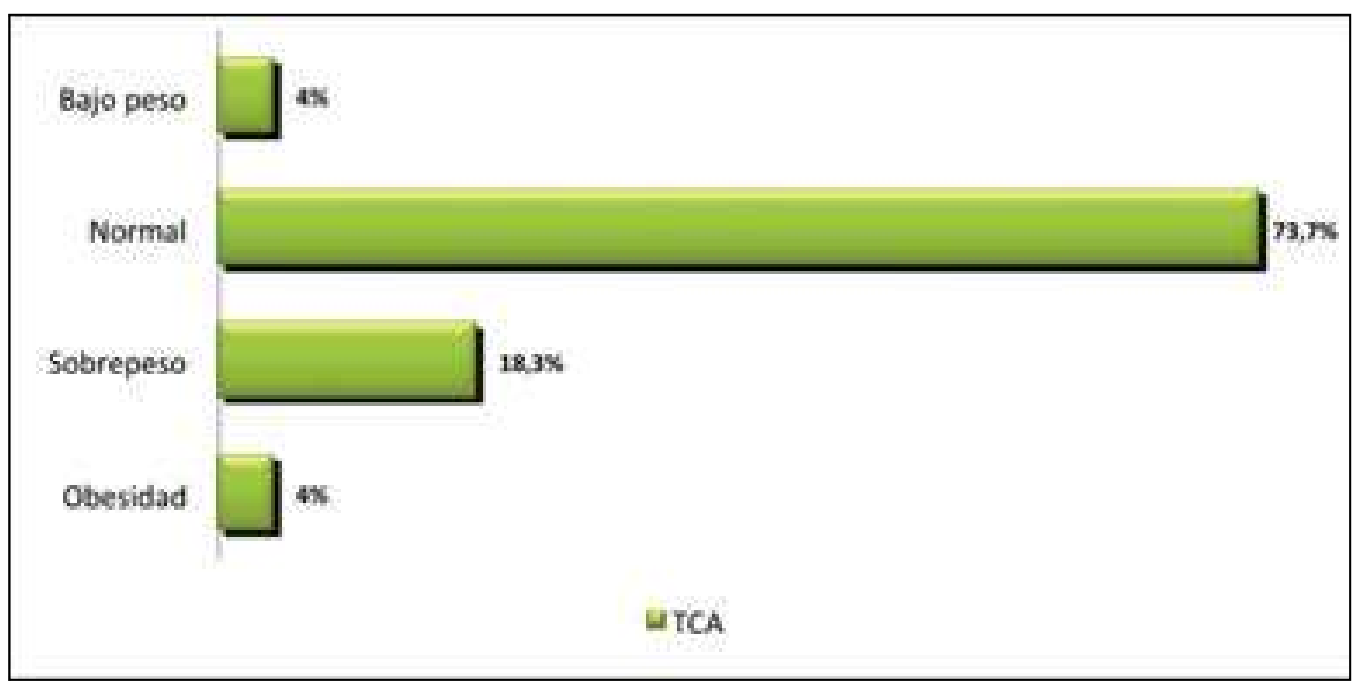

Fuente: Elaboración propia

Se observó mayor frecuencia de riesgo de TCA en los estudiantes de instituciones públicas $(31,3 \%)$ que en las privadas (28,7\%). (Gráfico 6)

Gráfico 6. Porcentaje de escolares con riesgo de TCA y tipo de Institución

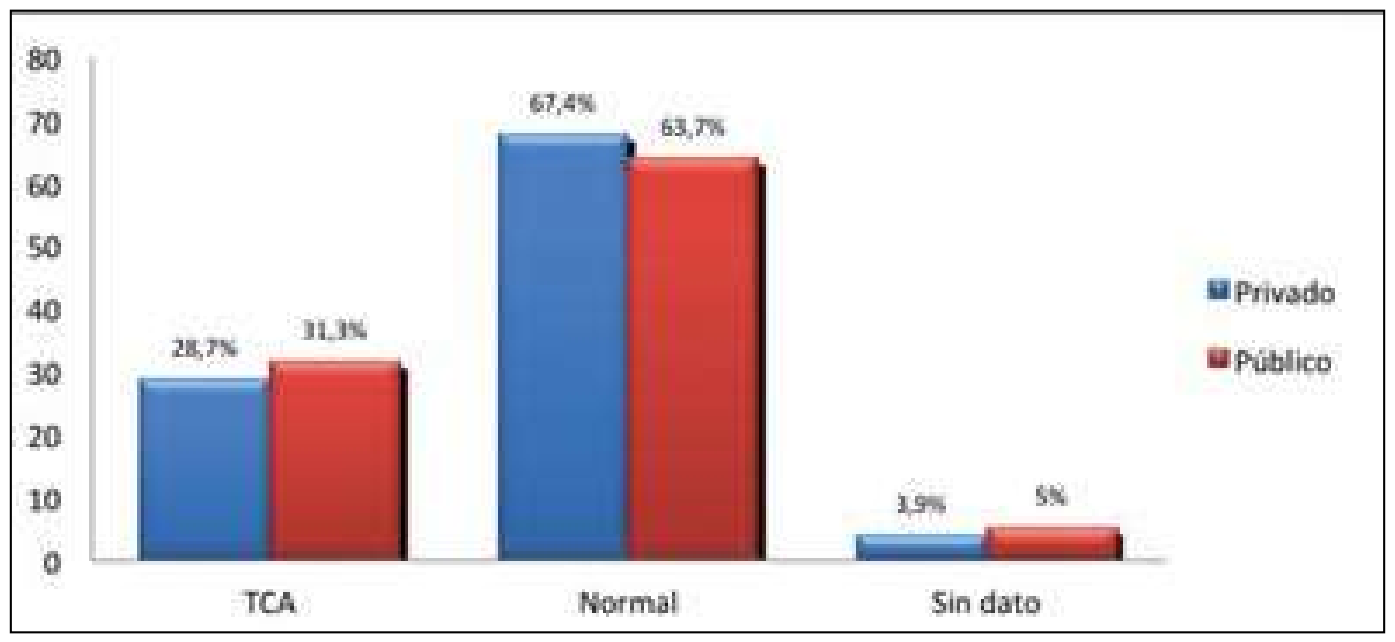

Fuente: Elaboración propia 


\section{Discusión}

La prevalencia de riesgo de trastornos alimentarios encontrada en este trabajo fue del $30,1 \%$, mayor a la reportada en investigaciones similares en el país. Sin embargo, cabe destacar que la prevalencia de TCA depende de las características de la población y del instrumento que se use para la medición del riesgo. Las escalas de riesgo de TCA se han estado evaluando y probablemente deben ser adaptadas a cada población según su contexto socio-cultural.

Se observó diferencias entre hombres y mujeres. Las mujeres presentan mayor prevalencia de TCA, datos consistentes con estudios previos que reportan mayor prevalencia en el grupo femenino (12-14). En diversas investigaciones internacionales en cuanto a los trastornos de la conducta alimentaria, se ha observado la percepción y las actitudes con relación al peso corporal encontrándose que las mujeres presentan mayor insatisfacción con el peso que los hombres; ellas quieren ser más delgadas aun teniendo un peso adecuado. Este factor sumado a la etapa de la adolescencia, representa un alto riesgo para desarrollar conductas alimentarias alteradas. (17)

En este grupo estudio también se observó que la mayoría de estudiantes con riesgo de TCA tienen un estado nutricional normal.

En esta población no se observaron diferencias con relación al tipo de institución educativa, aunque los resultados son similares a los reportados por Urzúa y Correa en Chile $(5,18)$ quienes encontraron mayor prevalencia de TCA en centros educativos públicos. Los autores refieren que dentro de las causas se encuentran la falta de control sobre su propia vida, auto desprecio e inseguridad.

Los hallazgos en cuanto al retraso del crecimiento son menores a los reportados en la Encuesta Nacional de la Situación Nutricional de Colombia (ENSIN) del 2010 (19) con un porcentaje de $10 \%$ y en cuanto al exceso de peso los resultados de este estudio son similares a los datos reportados a nivel nacional con un $17,5 \%$, lo cual preocupa ya que ha aumentado en un $25 \%$ aproximadamente en el último quinquenio.

La razón de la cintura/estatura demostró relacionarse con la clasificación nutricional por índice de masa corporal, lo cual indica aumento de grasa a nivel superior del cuerpo que acompañan al sobrepeso y la obesidad. Estos resultados son comparables con otras investigaciones a nivel nacional e internacional donde se relaciona el sobrepeso y la obesidad con el exceso de grasa abdominal, considerado riesgo cardiometabólico tanto en niños como en adultos $(20,21)$.

Los resultados de la frecuencia de consumo de alimentos son comparables con lo encontrado en la ENSIN 2010 (19) donde el 39\% de la población de 5 a 64 años no consume lácteos diariamente; se observó además un bajo consumo de carnes. Uno de cada 3 colombianos no consume frutas a diario y 5 de cada 7 no consume verduras en la misma frecuencia, resultados similares a los obtenidos en este estudio donde menos de la mitad de la población consume fruta entera y verduras diariamente refiriendo además el consumo de frutas en jugos primordialmente, lo cual disminuye el aporte de fibra dietaria.

En la población de estudiantes que no acostumbra desayunar o cenar se justificó esta conducta por la "falta de tiempo" o por no tener el hábito de tomar estas comidas. Estudios previos han demostrado que la mayoría de personas no consideran tener algún problema con su alimentación a menos que haya manifestaciones de deterioro del bienestar emocional o conductas inapropiadas como el vómito auto-inducido. Otros comportamientos no son considerados riesgos para la salud. Las personas que reconocen que hay alguna falencia en su consumo de alimentos son las más propensas a buscar orientación (22).

Programas de capacitación aplicados en escuelas afirman que el conocimiento sobre los trastornos de la conducta alimentaria, sus causas e implicaciones en el estado de salud permiten que las personas se sientan mejor preparadas para identificar las señales de riesgo desde una etapa temprana. Así mismo, permiten que se aumente la probabilidad de recuperación mediante la adquisición de hábitos alimentarios adecuados. Profesores y personal de las escuelas deben estar capacitados para desarrollar la habilidad de identificar a los estudiantes en riesgo y remitirlos para una intervención temprana y oportuna (23).

Debido a la importancia que tiene la imagen corporal, sobre todo en las edades donde la población es más vulnerable a este fenómeno, algunos estudios han propuesto llevar a cabo programas de promoción y prevención temprana para los TCA. Se busca que estas estrategias estén enfocadas a evitar la insatisfacción corporal y el uso de dietas reductoras de peso innecesarias. Así mismo, promover los hábitos saludables de alimentación y de ejercicio físico. Es así como se propone que familiares y amigos de población vulnerable deben estar capacitados y estar pendientes de ayudar a las personas con síntomas 
de TCA para actuar oportunamente dado que los TCA con frecuencia se presentan simultáneamente con otras condiciones médicas que pueden complicar su manejo, tales como episodios de depresión y/o ansiedad y problemas relacionados con el consumo de alcohol (24-26).

Dentro de las limitaciones de este estudio es un posible sesgo de la información en cuanto a la frecuencia de consumo de alimentos e historia nutricional, datos obtenidos por reporte de los estudiantes, dificultad que se describe en la literatura con respecto a estos métodos de recolección de datos.

\section{Conclusiones}

El riesgo para presentar Trastornos de la Conducta Alimentaria se observó en el $30,1 \%$ de los estudiantes que conformaron la muestra de este trabajo, siendo mayor la prevalencia de riesgo en las mujeres. Al relacionar este riesgo con el estado nutricional se observó mayor frecuencia en la población con índice de masa corporal dentro de los parámetros normales y en instituciones públicas.

Teniendo en cuenta que la presencia de Trastornos de la Conducta Alimentaria es cada vez más frecuente en la población mundial y que pueden desencadenar problemas cardíacos, renales e incluso la muerte, cobra interés el focalizar intervenciones preventivas o de acción tanto en instituciones educativas como para diseñar estrategias de salud pública que contribuyan al manejo de esta patología emergente en población infantil y juvenil.

\section{Conflicto de intereses}

Los autores declaran no tener de manera directa o indirecta, algún tipo de conflicto de intereses financieros, académicos o laborales que puedan poner en peligro la validez de este estudio.

\section{Agradecimientos y financiación}

Los autores agradecen al Estadístico Gerardo Ardila Duarte, por la revisión del análisis de datos.

A la Universidad Militar Nueva Granada por la aprobación y financiación del proyecto de investigación registrado como MED 1925.

\section{Referencias}

1. American Psychiatric Association. Diagnostic and Statical Manual of Mental Disorders, 4th edn. Washington, DC:
American Psychiatric Association 2000

2. American Dietetic Association. Position of the American Dietetic Association: Nutrition Intervention in the Treatment of Anorexia Nervosa, Bulimia Nervosa, and Other Eating Disorders. J Am Diet Assoc 2006; 106 (12):2073-2082.

3. Herpertz-Dahlmann B. Adolescent eating disorders: definitions, symptomatology, epidemiology and comorbidity. Child Adolesc Psychiatr Clin N Am 2009; 18(1):31-47.

4. Portela de Santana, M. L., da Costa Ribeiro Junior, H., Mora Giral, M., \& Raich, R. M. La epidemiología y los factores de riesgo de los trastornos alimentarios en la adolescencia: una revisión. Nutrición hospitalaria 2012; 27(2),391-401.

5. Urzúa, A., Castro, S., Lillo, A., \& Leal, C. Prevalencia de riesgo de trastornos alimentarios en adolescentes escolarizados del norte de Chile. Revista chilena de nutrición 2011, 38(2), 128-135.

6. Van Son GE, van Hoeken D, Bartelds AIM, van Furth EF, Hoek HW. Time trends in the incidence of eating disorders: A primary care study in The Netherlands. Int $J$ Eat Disord 2006; 39 (7):565-9.

7. Nicholls DE, Lynn R, Viner RM. Childhood eating disorders: British national surveillance study. $\mathrm{Br} \mathrm{J}$ Psychiatry 2011; 198(4):295-301.

8. Madden S, Morris A, Zurynski YA, Kohn M, Elliot EJ. Burden of eating disorders in 5-13-year-old children in Australia. Med J Aust 2009; 190 (8):410-4.

9. Peláez-Fernández MA, Raich RM, Labrador FG. Trastornos de la conducta alimentaria en España: Revisión de estudios epidemiológicos. Revista Mexicana de Trastornos Alimentarios 2010; 01 (01):62-75

10. Rueda, G. E., Díaz, L. A., Campo, A., Barros, J. A., Avila, G. C., Oróstegui. Validación de la encuesta SCOFF para tamizaje de trastornos de la conducta alimentaria en mujeres universitarias. Biomédica 2005, 25(2),196-202.

11. Cano AA, Castaño JJ, Corredor DA, et al. Factores de riesgo para trastorno de la alimentación en alumnos de Universidad de Manizales. Medunab. 2007; 10:187-94.

12. Fandiño A, Giraldo SC, Martínez C, et al. Factores asociados con los trastornos de la conducta alimentaria en estudiantes universitarios en Cali, Colombia. Colomb Med. 2007; 38:344-51.

13. Bustos-Leiton GJ, Romero-Chaparro A, Campo-Arias A. Asociación entre percepción de estrés y riesgo de trastorno de comportamiento alimentario. Salud Uninorte. 2011; 27:30-5.

14. Campo-Arias, A., \& Villamil-Vargas, M. (2012). Riesgo de trastorno del comportamiento alimentario (TCA) en estudiantes de medicina en Colombia. Revista colombiana de Psiquiatría 2012, 41(2), 328-339 
15. Morgan, J. F., Reid, F. \& Lacey, J. H. (1999). The SCOFF questionnaire: assessment of a new screening tool for eating disorder. British of Medicine Journal, 319,1467-1468.

16. Campo-Arias, A., Díaz-Martínez, L. A., Rueda-Jaimes, G. E., Martinez-Mantilla, J. A., Amaya-Naranjo, W., \& Campillo, H. A. Consistencia interna y análisis factorial del cuestionario SCOFF para tamizaje de trastorno de conducta alimentaria en adolescentes estudiantes: Una comparación por género. Universitas Psychologica 2006; 5(2),295-304.

17. Cruzat-Mandich C., Díaz Castrillón F., Lizana-Calderón P., Castro A. Comparación por sexo en imagen corporal, síntomas psicopatológicos y conductas alimentarias en jóvenes entre 14 y 25 años. Rev Med Chile 2016; 144: 743-750

18. Correa V. Zubarew G. Silva P Romero M. Prevalencia de riesgo de trastomos alimentarios en adolescentes mujeres escolares de la Region Metropolitana. Rev Chil Pediatr 2006; 77:153-60.

19. Ministerio de Protección Social, Instituto Colombiano de Bienestar Familiar, Instituto Nacional de Salud. Encuesta Nacional de la Situación Nutricional en Colombia 2010 ENSIN. Bogota: Oficina Asesora de Comunicaciones y Atención al Ciudadano ICBF; 2011. P. 1-509

20. Fajardo E., Arango L. Prevalencia de sobrepeso y obesidad, consumo de alimentos y patrón de actividad física en una población de niños escolares de la ciudad de Bogotá. Rev Med 2012; 20(1):101-116.

21. Carmenate M, Marrodán M, Mesa M, González M, Alba J. Obesidad y circunferencia de la cintura en adolescentes madrileños. Rev Cubana Salud Pública. 2007; 33(3): 1019.

22. Gratwick-Sarll K., Bentley C., Harrison C., Mond, J. Poor self-recognition of disordered eating among girls with bulimic-type eating disorders: cause for concern. Early intervention in psychiatry 2016; 10 (4): 316-323.

23. Anderson C., Holody K., Flynn L., HussaFarrell R. An exploratory evaluation of the feasibility, acceptability, and efficacy of the mental fitness disordered eating program in schools. Eating Disorders 2017; 25 (3): 230-245

24. Keski-Rahkonen A., Mustelin L. Epidemiology of eating disorders in Europe: prevalence, incidence, comorbidity, course, consequences, and risk factors. Curr Opin Psychiatry 2016, 29:340-345

25. Vaquero-Cristóbal R., Alacid F., Muyor J., López-Miñarro P. Imagen corporal; revisión bibliográfica. Nutr Hosp. 2013;28(1):27-35

26. McAndrew A., Menna R. Perceptions of disordered eating and associated help seeking in young women. Eating Disorders 2017. Disponible en: http://dx.doi.org/10.1080/ 10640266.2017.1318624 